
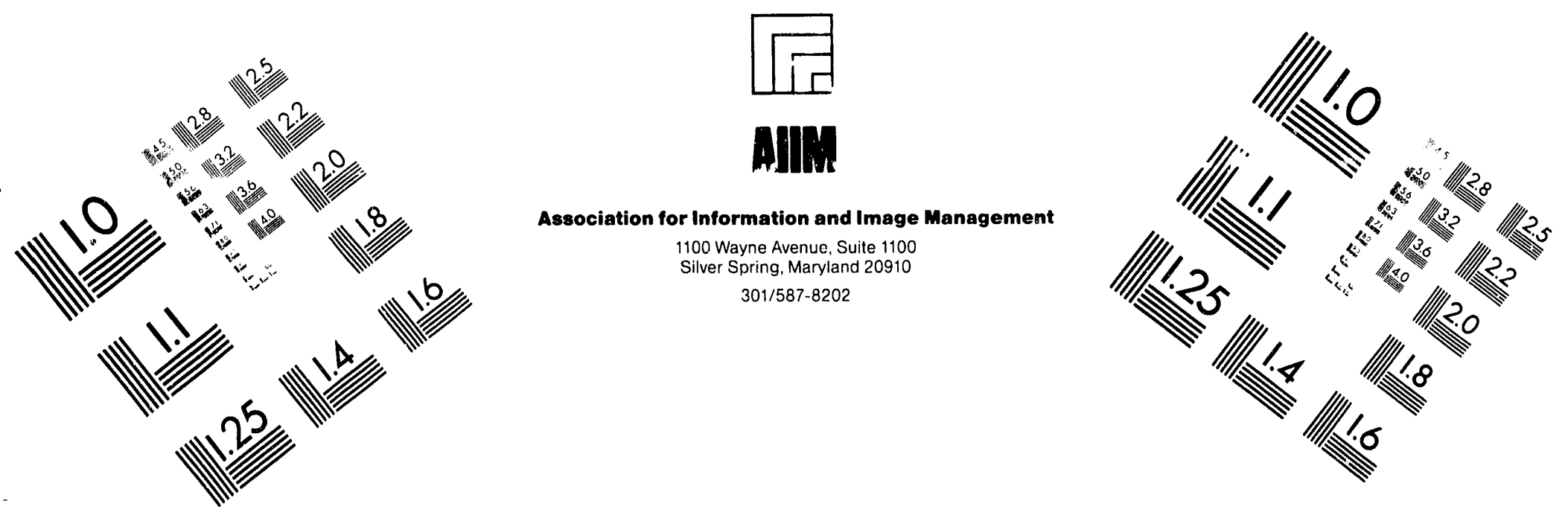

\title{
Centimeter
}

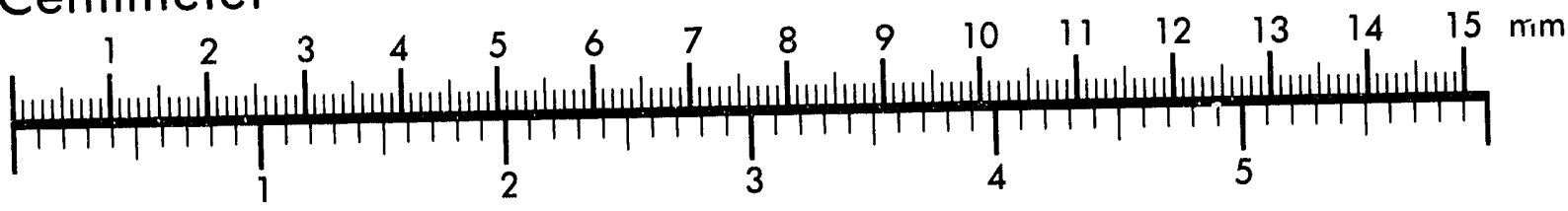
Inches
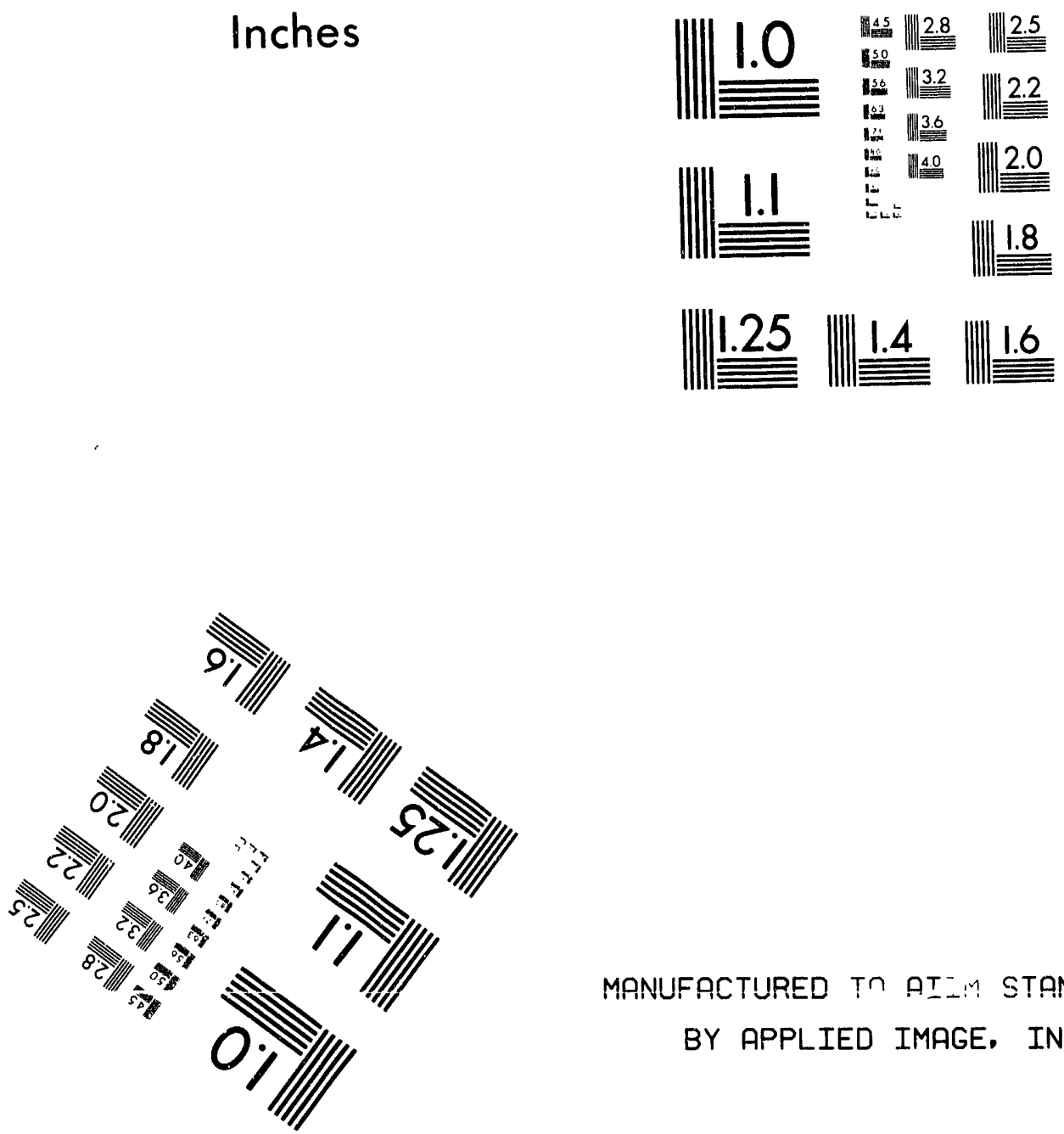

MANUFACTURED TO RT-M STANDARDS

BY APPLIED IMAGE, INC.

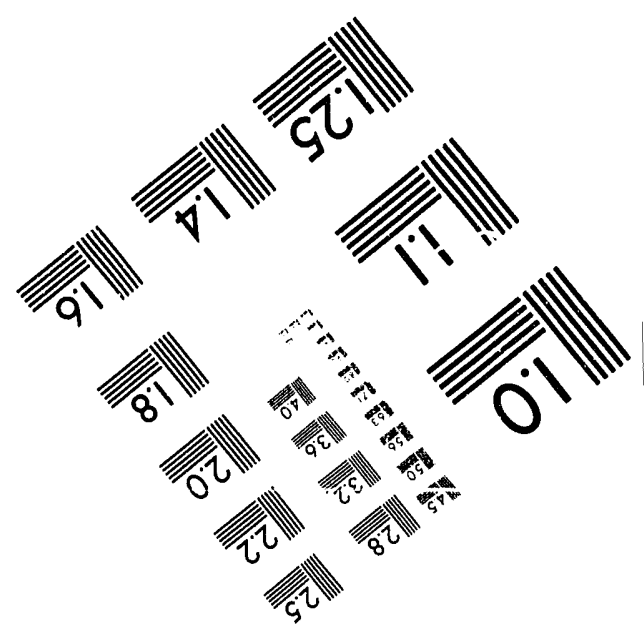



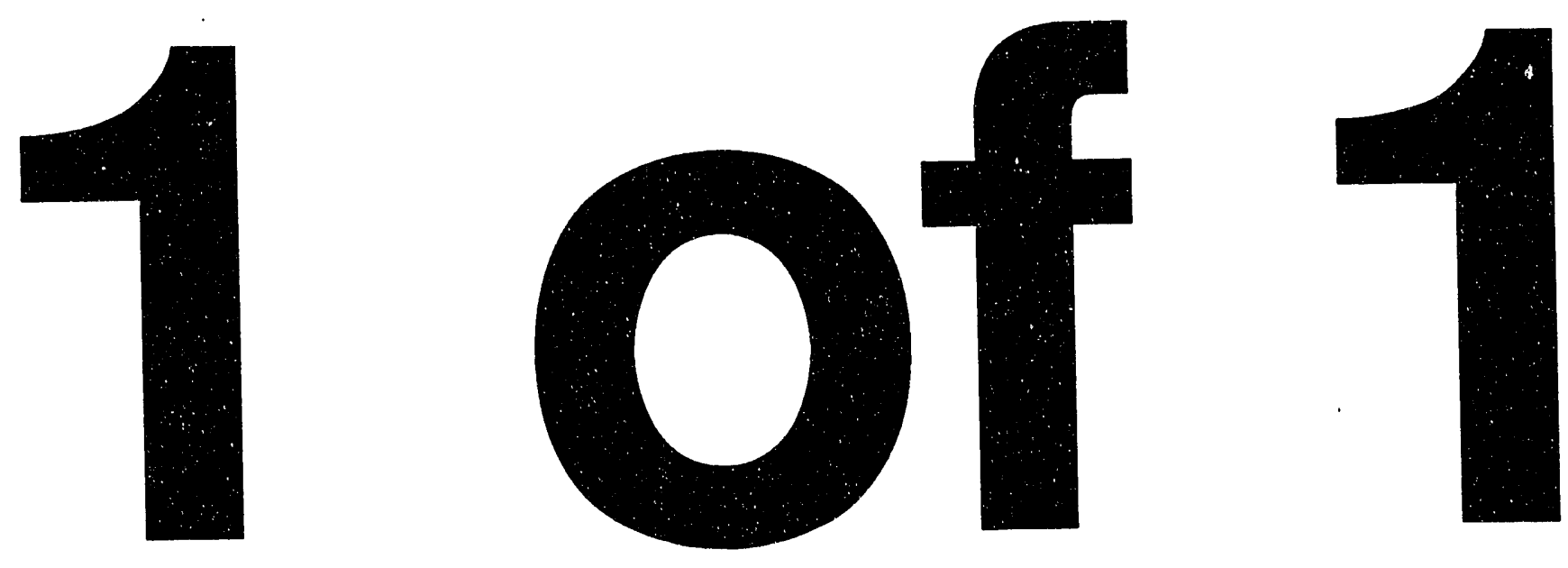
$\frac{48}{6}-9398$ (2)

PREPARED FOR THE U.S. DEPAR IMENT OF ENERGY, UNDER CONTRACT DE-AC02-76-CHO-3073

PPPL-2907

UC-421,427

\section{THEORY OF KINETIC BALLOONING MODES EXCITED BY ENERGETIC PARTICLES IN TOKAMAKS}

BY

S. TSAI AND L. CHEN

\author{
MAY, 1993
}

paINCETON

plasma phrgics

LABOAATOAY

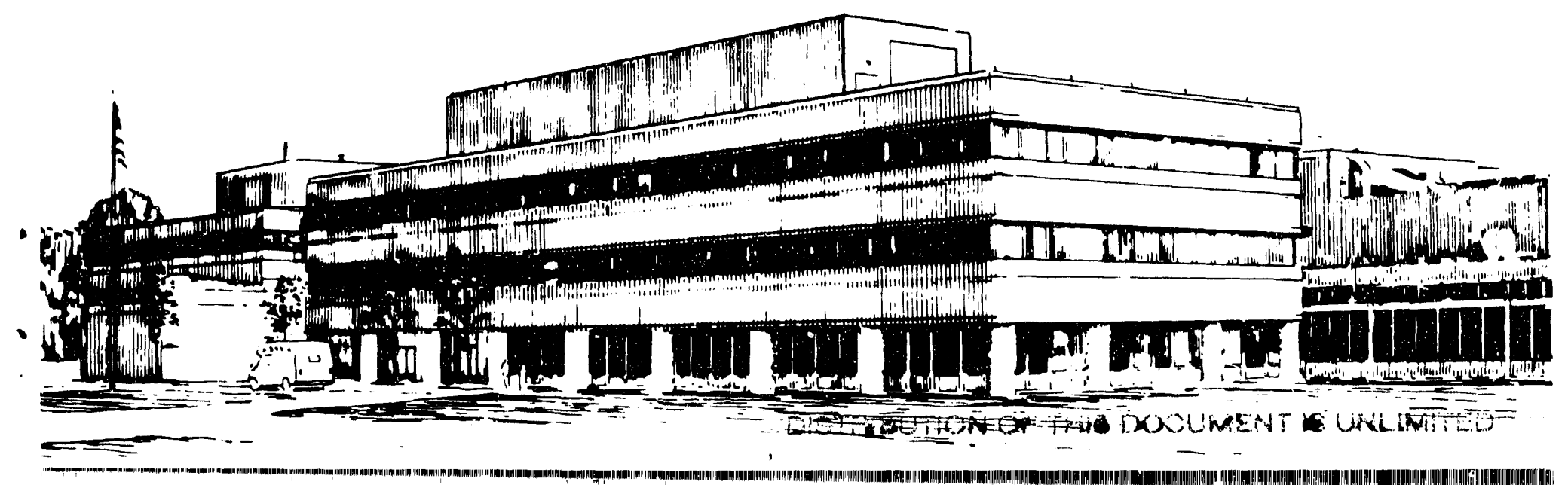




\section{NOTICE}

This report was prepared as an account of work sponsored by an agency of the United States Government. Neither the United States Government nor any agency thereof, nor any of their employees, makes any warranty, express or implied, or assumes any legal liability or responsibility for the accuracy, completeness, or usefulness of any information, apparatus, product, or process disclosed, or represents that its use would not infringe privately owned rights. Reference herein to any specific commercial produce, process, or service by trade name, trademark, manufacturer, or otherwise, does not necessarily constitute or imply its endorsement, recommendation, or favoring by the United States Government or any agency thereof. The views and opinions of authors expressed herein do not necessarily state or reflect those of the United States Government or any agency thereof.

\section{NOTICE}

This report has been reproduced from the best available copy.

Available in paper copy and microfiche.

Number of pages in this report: 20

DOE and DOE contractors can obtain copies of this report from:

Office of Scientific and Technical Information

P.O. Box 62

Oak Ridge, TN 37831;

(615) 576-8401.

This report is publicly available from the:

National Technical Information Service

Department of Commerce

5285 Port Royal Road

Springfield, Virginia 22161

(703) $487-4650$ 


\title{
Theory of Kinetic Ballooning Modes Excited by Energetic Particles in Tokamaks
}

\author{
Shih-Tung Tsai* \\ and \\ Liu Chen \\ Princeton University, Plasma Physics Laboratory \\ and \\ Department of Astrophysical Sciences \\ P.O. Box 451 \\ Princeton, N.J. 08543
}

\begin{abstract}
We have analyzed theoretically the resonant excitations of kinetic ballooning modes (KBM) by the energetic ions/alpha particles in tokamaks. Our theory includes finite-size orbit effects of both circulating and trapped particles. With energetic-particle contributions suppressed in the singular inertial layer, an analytic. dispersion relation can then be derived via an asymptotic matching analysis. The dispersion relation, in particular, demonstrates the existence of two types of modes; that is, the magnetohydrodynamic ( $M H D$ ) gap mode and the energetic-particle continuum mode. Specific expressions for real frequencies, growth rates and threshold conditions are also derived for a model slowing-down beam ion distribution function.
\end{abstract}

"permanent address: Inst. of Physics, Chinese Academy of Sciences, Beijing 100080. PRC 


\section{Introduction}

The dynamics of energetic ions/ $\alpha$ particles is an important issue for fusion research and has attracted, in recent years, increasing experimental ${ }^{1-3}$ as well as theoretical ${ }^{4-13}$ attention. There exists a considerable amount of works devoted to the study of how the energetic particles affect the stability of MHD modes. In particular, it has been demonstrated that MHD modes can be driven unstable via resonances with energetic particles. ${ }^{7-13}$ Most of previous theoretical analyses have assumed that the energetic particles have negligible orbit sizes. In the presence of sharply localized singular-layer structures, this assumption is, at best, dubious. Only recently, the stabilizing effects of finitesize orbits have been included in the analyses of toroidal Alfvén eigenmodes (TAE). ${ }^{14,15}$

In this paper, we examine finite-size orbit effects of energetic particles on KBM instabilities. Noting that KBM consists of a singular inertial and a regular ideal regions, the finite-size orbits then render energetic particles effective only in the ideal region. Employing an asymptotic matching analysis, a general dispersion relation can then be derived analytically for both circulating and trapped particles; including corresponding various wave-particle resonances. This dispersion relation has the generic form of the "fishbone" dispersion relation, ${ }^{7-9}$ and demonstrates, in particular, the existence of two types of modes. ${ }^{8}$ One is the MHD gap mode which has a frequency in the order of ion diamagnetic drift frequency and can be destabilized by the energetic particles only when the plasma is ideal MHD unstable. The other is the energetic-particle continuum mode; which, however, has a threshold condition and the frequency corresponds to that of energetic-particle characteristic frequency.

Section II presents the theoretical model and the relevant eigenmode equations. The corresponding dispersion relation is then obtained via asymptotic matching in Sec. III. In Sec. IV, we analyze the general stability properties of KBM and derive contributions due to circulating and trapped energetic particles. Specific results of a model slowing-down beam ion distribution function are given in Sec. V. Finally, Sec. VI contains a brief summary and discussions. 


\section{Theoretical Model and Eigenmode Equations}

We consider a large-aspect ratio (i.e. $\epsilon=a / R_{0} \ll 1$ ) tokamak plasma consisting of a warm core (C) component and an energetic (E) component. Here, $a$ and $R_{0}$ are, respectively, the minor and major radii. In terms of the smallness parameter $\epsilon$, we adopt the following formal orderings: $\beta_{C} \equiv$ $8 \pi P_{C} / B^{2} \sim \epsilon, \beta_{E} \sim \epsilon^{2}, T_{C} / T_{E} \sim \epsilon^{2}, n_{E} / n_{C} \sim \epsilon^{3}, k_{\theta} \rho_{i C} \sim \epsilon^{3 / 2}, k_{\theta} \rho_{E} \sim \epsilon^{1 / 2}$, and $\omega / \omega_{A} \sim \epsilon$. Here, $\omega_{A}=V_{A} / q R_{0}, q$ is the safety factor and the rest of the notations is standard. We note that the present orderings are adopted such that effects of finite-size orbits, while suppress energetic-particle dynamics in the inertial layer, affect the core component negligibly. Furthermore, to make analytical calculations of the KBM with finite $k_{\theta} \rho_{E}$ possible, we adopt the Connor-Hastie-Taylor analytical model equilibrium of circular magnetic surfaces. ${ }^{16}$

Within the present model, the ideal MHD approximation can be shown to remain valid; i.e. $\delta E_{\|} \simeq 0$. Meanwhile, perpendicular pressure balance gives

$$
B \delta B_{\|} \simeq 4 \pi n_{i} e \omega_{* p} \frac{\delta \phi}{\omega},
$$

where $n_{i} e \omega_{* p} \equiv(c / B)\left(\mathbf{k} \times \mathbf{e}_{\|}\right) \cdot \nabla P$, and $\delta \phi$ is the scalar potential. The eigenmode equation then corresponds to the following vorticity equation:

$$
\left[\frac{d}{d \theta} f \frac{d}{d \theta}+\Omega^{2} f+\alpha g\right] \delta \phi-\sum_{j} \eta_{j}<g \Omega_{d} \cdot J_{0}\left(k_{\perp} \rho\right) \delta G>_{j}=0
$$

where we have used the well-known ballooning mode representation. Thus, $\theta$ is the extended coordinate along the magnetic field $\mathbf{B}, \Omega^{2} \equiv \omega(\omega-$ $\left.\omega_{* p_{i}}\right) / \omega_{A}^{2}, f(\theta)=1+(s \theta-\alpha \sin \theta)^{2}, s=r q^{\prime} / q, \alpha=q^{2} R_{0} \beta^{\prime}, g(\theta)=\cos \theta+$ $(s \theta-\alpha \sin \theta) \sin \theta, \eta_{j}=4 \pi e_{j} q^{2} R_{0}^{2} \omega / c^{2}, \omega_{d j}(\theta)=k_{\theta} \Omega_{d j} g(\theta), \Omega_{d j}=\left(v_{\perp}^{2} / 2+\right.$ $\left.v_{\|}^{2}\right) / \omega_{c j} R_{0}$, and $\langle\cdots\rangle_{j} \equiv \int d^{3} v(\cdots)_{j}$ with $j$ being the relevant species. The function $\delta G_{j}$ corresponds to the nonadiabatic gyrokinetic response and is governed by the following gyrokinetic equation:

$$
\left[\omega_{t} \frac{d}{d \theta}-i\left(\omega-\omega_{d}\right)\right]_{j} \delta G_{j}=i\left(\frac{e}{m}\right)_{j} Q F_{o j} g \Omega_{d j} J_{0}\left(\lambda_{\rho},\right) \frac{\delta \phi}{\omega},
$$

where $\omega_{t}=v_{\|} / q R_{0}, Q F_{o j}=\left(\omega \partial_{\mathcal{E}}+\hat{\omega}_{*}\right) F_{o j}, \mathcal{E}=v^{2} / 2, \lambda_{\rho}=k_{\perp} \rho$, and $\hat{\omega}_{*} F_{o j}=\left(\mathbf{k} \times \mathbf{e}_{\|} / \omega_{c j}\right) \cdot \nabla F_{o j}$. Note that, in Eq. (3), the left-hand side stands 
for the guiding-center propagator and, hence, determines the wave-particle resonance conditions, $Q F_{o j}$ corresponds to the free energy in both configuration and velocity spaces, and $\Omega_{d j} \delta \phi$ indicates energy exchange via $V_{d j} \cdot \delta E$ with $V_{d j}$ being the magnetic drift.

For the present analysis, it is more convenient to let $\delta \psi=f^{1 / 2} \delta \phi$ and rewrite Eq. (2) in the following standard form:

$$
\left[\frac{d^{2}}{d \theta^{2}}+\Omega^{2}+\frac{\alpha \cos \theta}{f}-\frac{(s-\alpha \cos \theta)^{2}}{f^{2}}\right] \delta \psi-f^{-1 / 2} \sum_{j} \eta_{j}\left\langle g \Omega_{d} J_{0}\left(\lambda_{\rho}\right) \delta G>_{j}=0 .\right.
$$

Equations (3) and (4) correspond to the coupled differential-integral eigenmode equations and are generally difficult to solve analytically. Significant progress, however, can be made by noting that the basic mode structures contain two spatial regions; that is the long-scale $\left[\theta \sim O\left(\omega_{A} / \omega\right) \sim \epsilon^{-1}\right]$ inertial and the short- scale $[\theta \sim O(1)]$ ideal regions. Analytical dispersion relation can then be derived by asymptotically matching the solutions in the two regions, and the details are given in the next Section.

\section{Dispersion Relation}

In the inertial region, we have $|\theta| \sim O\left(\epsilon^{-1}\right) \gg 1$. It can then be straightforwardley demonstrated that, with the formal $\left|k_{\theta} \rho_{E}\right| \sim \epsilon^{1 / 2}$ ordering, energetic-particle nonadiabatic contributions are negligible due to the finite size orbit effects. Core particle contributions, meanwhile, remain essentially the same as the negligible orbit-size case ${ }^{12,13}$ and, hence, will be omitted in the present analysis. Equation (4), therefore, reduces to

$$
\left(\frac{d^{2}}{d \theta^{2}}+\Omega^{2}\right) \delta \psi_{0}(\theta)=0
$$

The solution is

$$
\delta \psi_{0}(\theta)=e^{i \Omega|\theta|},
$$

with the $\operatorname{Im} \Omega>0$ causality constraint.

In the ideal region, meanwhile, we have $|\theta| \sim O(1)$, and Eq. (4) reduces to

$$
\left[\frac{d^{2}}{d \theta^{2}}+\frac{\alpha \cos \theta}{f}-\frac{(\alpha \cos \theta-s)^{2}}{f^{2}}\right] \delta \psi_{I}-f^{-1 / 2} g \eta_{E}<\Omega_{d} J_{0} \delta G>_{E}=0 .
$$




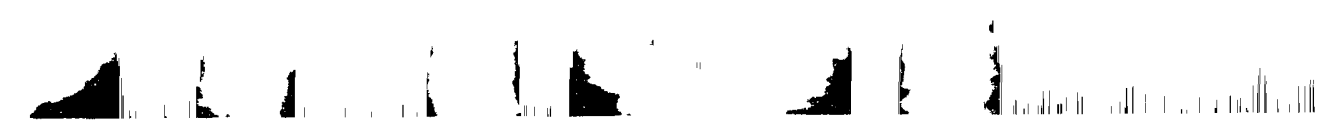

From Eq. (7), we can construct the following quadratic form

$$
\begin{aligned}
\left.\delta \psi_{I}^{*} \frac{d \psi_{I}}{d \theta}\right|_{-\infty} ^{\infty} & +\int_{-\infty}^{\infty} d \theta\left[-\left|\frac{d \delta \psi_{I}}{d \theta}\right|^{2}+\left(\frac{\alpha \cos \theta}{f}-\frac{(\alpha \cos \theta-s)^{2}}{f^{2}}\right)\left|\delta \psi_{I}\right|^{2}\right] \\
& -\int_{-\infty}^{\infty} d \theta f^{-1 / 2} g \eta_{E} \delta \psi_{I}^{*}<\Omega_{d} J_{0} \delta G>_{E}=0 .
\end{aligned}
$$

Asymptotically matching $\delta \psi_{I}$ and $\delta \psi_{0}$ gives

$$
\left.\delta \psi_{I}^{*} \frac{d}{d \theta} \delta \psi_{I}\right|_{-\infty} ^{\infty}=2 i \Omega
$$

Substituting Eq. (y) into Eq. (8) yields the desired dispersion relation

$$
-i \Omega+\delta W_{f}+\delta W_{K}=0
$$

where $\delta W_{f}$ is the well-known MHD fluid $\delta W$, i.e.,

$$
\delta W_{f}=\frac{1}{2} \int_{-\infty}^{\infty} d \theta\left\{\left|\frac{d \delta \psi_{I}}{d \theta}\right|^{2}+\left[\frac{(s-\alpha \cos \theta)^{2}}{f^{2}}-\frac{\alpha \cos \theta}{f}\right]\left|\delta \psi_{I}\right|^{2}\right\}
$$

$\delta W_{K}$ is the kinetic $\delta W$ due to the energetic-particle "compression", i.e.,

$$
\delta W_{K}=\frac{1}{2} \eta_{E} \int_{-\infty}^{\infty} d \theta<\delta S^{*} \delta G>_{E}
$$

and

$$
\delta S=\Omega_{d} f^{-1 / 2} g J_{0}\left(\lambda_{\rho}\right) \delta \psi_{I}
$$

Note that the causality constraint requires

$$
\delta W_{f}+\delta W_{K r}<0
$$

with $\delta W_{K r} \equiv \operatorname{Re}\left(\delta W_{K}\right)$. It is also interesting to note that the dispersion relation, Eq. (10), has the same form as that of the "fishbone" instability. ${ }^{7-9}$ We therefore, term it as the generic "fishbone" dispersion relation. 


\section{General Stability Analyses}

Since we are interested in the energetic particle-wave resonances, we let

$$
\delta W_{K}=\delta W_{K r}+i \delta W_{K i}
$$

Furthermore, we let $\omega=\omega_{r}+i \gamma$ with $\left|\gamma / \omega_{r}\right| \ll 1$ for near marginal stability studies.

From Eq. (10), it is clear that the stability properties depend crucially on $\Omega$. Noting that $\Omega^{2} \equiv \omega\left(\omega-\omega_{* p_{i}}\right) / \omega_{A}^{2}$, we can then identify two types of modes $^{8}$ depending on the sign of $\Omega^{2}\left[\omega=\omega_{r}\right]$. Specifically, for

$$
\omega_{r}<0 \text { or } \omega_{r}>\omega_{* p i},
$$

we have $\Omega$ being real and, hence, the continuum mode. On the otherhand, for $\omega_{r}$ inside the $\left(0, \omega_{* p_{i}}\right)$ ion diamagnetic gap, we have $\Omega$ being imaginary and, hence, the gap mode. In the following, we shall discuss the stability properties of these two types of modes separately.

For the continuum mode, $\omega_{r}$ is determined by the real part of dispersion relation, Eq.(10); i.e.,

$$
\delta W_{f}+\delta W_{K r}=-\gamma \frac{\partial \Omega_{r}}{\partial \omega_{r}}<0 .
$$

The growth rate, meanwhile, is given by the imaginary part of the dispersion relation and can be written as

$$
\gamma=\left(\delta W_{K i}-\Omega_{r}\right) /\left(-\frac{\partial \delta W_{K r}}{\partial \omega_{r}}\right)
$$

The threshold condition is, thus,

$$
\delta W_{K i}=\Omega_{r}
$$

i.e., balancing the energetic-particle drive with the continuum damping. For the mode to be unstable, it requires $\delta W_{K_{i}}>\Omega_{r}$, and

$$
\partial \delta W_{K^{\prime}} / \partial \omega_{r}<0 .
$$

Since this mode is induced by the energetic particles, we shall further term it as the energetic particle continuum mode. 
For the gap mode, meanwhile, the dispersion relation, Eq. (10), can be rewritten as

$$
\Omega^{\prime}+\delta W_{f}+\delta W_{K}=0
$$

where $\Omega^{\prime} \equiv-i \Omega=\sqrt{\omega\left(\omega_{* p i}-\omega\right)} / \omega_{A}$. The real part of Eq. (21), which determines $\omega_{r}$, is

$$
\Omega_{r}^{\prime}+\delta W_{f}+\delta W_{K r}=0
$$

where the causality constraint implies $\Omega_{r}^{\prime}>0$. The imaginary part of the dispersion relation then gives rise to the growth rate

$$
\gamma \simeq \frac{2 \delta W_{K i}\left(\delta W_{j}+\delta W_{K r}\right)}{\omega_{* p i}-2 \omega_{r}} \omega_{A}^{2} .
$$

Noting that $\delta W_{f}+\delta W_{K_{r}}<0$ and $\delta W_{K_{i}}$ is positive definite, the gap mode can be driven unstable only when

$$
\omega_{* p i}>\omega_{r}>\omega_{* p i} / 2 .
$$

Note that the gap mode is basically a MHD mode, and hence, will be called as the MHD gap mode.

In the rest of this Section, we are going to derive specific expressions of $\delta G$ and, hence, $\delta W_{K}$ for circulating and trapped particles. To do this, we need to know $\delta \phi_{I}\left(\delta \psi_{I}\right)$. Observing that, with $\delta W_{K} \sim \epsilon$, the lowest-order $\delta \psi_{I}$ is sufficient for the present purpose. If we further assume $s \sim \alpha<1$, it is then reasonable to adopt $\delta \psi_{\mathrm{I}}=1$.

\section{A. Circulating particle contribution}

It is more convenient to analyze the circulating particles via a straightforward transformation from the guiding centers to the drift centers. $\delta W_{k}$, Eq. (12), then becomes

$$
\delta W_{K}=\frac{2 \pi e q^{2} R_{0}^{2}}{c^{2}} \omega \int_{-\infty}^{\infty} d \theta<\delta S_{d}^{*} \delta G_{d}>
$$

where $\theta \sim O\left(\theta_{k} \sim 1 / s k_{\theta} \rho_{E}\right) \sim \epsilon^{-1 / 2}>1, \delta G_{d}=\delta G \exp \left(i \theta_{d}\right), \theta_{d}=-\lambda_{d} \cos \theta$, $\lambda_{d}=k_{\perp} \rho_{d}=k_{\perp} \Omega_{d} / \omega_{t}$, and

$$
\delta S_{d}^{*}=\Omega_{d} f^{-1 / 2} g J_{0}\left(\lambda_{\rho}\right) \exp \left(-i \theta_{d}\right)
$$




\section{4}

$\delta G_{d}$ is given by the following drift-center gyrokinetic equation

$$
\left(\omega_{t} \partial_{\theta}-i \omega\right) \delta G_{d}=i \frac{e}{m} \frac{Q F_{0}}{\omega} \Omega_{d} J_{0}\left(\lambda_{\rho}\right) \sin \theta_{0} \exp \left(i \theta_{d}\right) .
$$

Here, we have explicitly denoted the subsidiary two scales; $\theta_{0} \sim 0(1)$ and $\theta_{k} \sim O\left(\epsilon^{-1 / 2}\right)$. Noting that $\partial_{\theta} \simeq \partial_{\theta_{0}}$ and

$$
\Omega_{d} J_{0} \sin \theta_{0} e^{i \theta_{d}}=\Omega_{d} J_{0} \sum_{n=1}^{\infty} 2(-1)^{n} i^{n+1} \frac{n J_{n}\left(\lambda_{d}\right)}{\lambda_{d}} \sin n \theta_{0} \equiv \sum_{n=1}^{\infty} \delta S_{d n} \sin n \theta_{0},
$$

Eq. (27) can then be readily solved and we obtain

$$
\delta G_{d}=\frac{e}{m} Q F_{0} \sum_{n=1}^{\infty} \frac{\delta S_{d n}}{n^{2} \omega_{t}^{2}-\omega^{2}}\left(\sin n \theta_{0}-i \frac{n \omega_{t}}{\omega} \cos n \theta_{0}\right) .
$$

Equation (29) into Eq. (25), $\delta W_{K}$ for the circulating (untrapped) energetic particles then becomes

$$
\delta W_{K U}=\frac{\pi e^{2} q^{2} R_{0}^{2} \omega}{m c^{2}} \sum_{n=1}^{\infty}\left\langle\frac{Q F_{0}}{n^{2} \omega_{t}^{2}-\omega^{2}} \int d \theta\left|\delta S_{d n}\right|^{2}\right\rangle .
$$

Here, $\left|\delta S_{d n}\right|=2 \Omega_{d} J_{0}\left(\lambda_{\rho}\right) n J_{n}\left(\lambda_{d}\right) / \lambda_{d}$ and we have dropped the subsript $E$ to simplify the notations.

Recalling that, from Eq. (19), we have threshold $\delta W_{K i}=\Omega_{r} \simeq \omega_{r} / \omega_{A}$ for the energetic-particle continuum mode and, from Eq. (24), $\omega_{r} \sim \omega_{s p i}<\omega_{t E}$ for the MHD gap mode, we can then conclude that, in general cases, only the $n=1$ term in Eq. (30) needs to be considered. Adopting Padé's approximant for the Bessel functions; i.e.,

$$
J_{0}\left(\lambda_{\rho}\right) 2 J_{1}\left(\lambda_{d}\right) / \lambda_{d} \simeq\left(1+\Delta^{2} k_{\perp}^{2} / k_{\theta}^{2}\right)^{-1}
$$

where $\Delta^{2} \equiv k_{\theta}^{2}\left(\rho^{2}+\rho_{d}^{2} / 2\right) / 4$, Eq. (30) then becomes, keeping only the $n=1$ term,

$$
\delta W_{K U} \simeq \frac{\pi^{2}}{2} \frac{e^{2} q^{2} R_{0}^{2}}{m c^{2} s}\left\langle\frac{Q F_{0} \Omega_{d}^{2}}{\Delta\left(1+\Delta^{2}\right)^{3 / 2}} \frac{\omega}{\omega_{t}^{2}-\omega^{2}}\right\rangle
$$

We note also that, in deriving Eqs. (29) and (30), we have assumed $\partial_{\theta}=\partial_{\theta_{0}}$ and, hence, ignored the $\theta_{k}$-scale variations of $\lambda_{\rho}, \lambda_{d}$ and, hence, 
$\delta S_{d n}$. Keeping the $O\left(\epsilon^{1 / 2}\right) \partial_{\theta_{k}}$ corrections leads to broadening of waveparticle resonance conditions due to finite-size orbits. Specifically, Eq. (32) is modified (see Appendix) to become

$$
\begin{aligned}
\delta W_{K U} & =\frac{\pi^{2}}{4} \frac{e^{2} q^{2} R_{0}^{2}}{m c^{2}}\left\langle\frac{Q F_{0} \Omega_{d}^{2}}{\Lambda\left(1+\Delta^{2}\right)^{2}} \int_{0}^{\infty} d x e^{-x}\right. \\
& {\left.\left[\frac{1}{\omega_{t}-\omega+(\Lambda / 2) \omega_{t} x}-\frac{1}{\omega_{t}+\omega-(\Lambda / 2) \omega_{t} x}\right]\right\rangle }
\end{aligned}
$$

where $\Lambda=s \Delta /\left(1+\Delta^{2}\right)^{1 / 2}$. Note that the finite- $\Lambda$ resonance broadening effects could, in principle, also enhance the instability drive.

\section{B. Trapped Particle Contribution}

Similar to the circulating particles, the kinetic contribution of the trapped energetic particles can be more readily derived in terms of banana centers as, omitting the subscript $E$,

$$
\delta W_{K T}=2 \pi \eta B_{0} \int d \mathcal{E} \int d \mu \sum_{l=-\infty}^{\infty} \oint \frac{d \theta}{\left|v_{\|}\right|} \delta S_{b}^{*} \delta G_{b}
$$

where

$$
\delta S_{b}^{*} \equiv \Omega_{d} f^{-1 / 2} g J_{0}\left(\lambda_{\rho}\right) \exp \left(-i \theta_{b}\right),
$$

$\theta_{b}=\int^{\theta}\left(\omega_{d}-\bar{\omega}_{d}\right) d \tau, d \tau=q R_{0} d \theta /\left|v_{\|}\right|, \bar{A} \equiv \oint A d \tau / \tau_{b}, \tau_{b}=\oint d \tau, k_{\perp}^{2}=1+$ $(2 \pi l+\theta)^{2} s^{2}$, and $\delta G_{b}$ is the solution of gyrokinetic Eq. (3) with $\delta G_{b}=\delta G e^{i \theta_{b}}$. The subscript $b$, thus, denotes the banana centers.

While one can substitute the known solutions of $\delta G$ or $\delta G_{b}$ (see Ref. 17) into Eq. (34) to derive a formal expression of $\delta W_{K T}$, we shall try to further simplify the analysis by assuming the energetic particles are deeply trapped between $2 \pi l \pm \theta_{T}$. We then derive, for $|\omega| \sim\left|\bar{\omega}_{d E}\right|<\left|\omega_{b E}\right|$,

$$
\delta W_{K T} \simeq \frac{8 \pi^{2} e^{2} q R_{0} B_{0}}{m c^{2}} \int d \mathcal{E} \int d \mu Q F_{0} \Omega_{d}^{2} \tau_{b}\left(\frac{1}{\bar{\omega}_{d}-\omega}\right) .
$$

In deriving Eq. (36), we have noted that, compared to the $l=0$ term, $l \neq 0$ terms are negligible due to the $f^{-1}$ factor. Note that only physics of the precessional drift is kept here. ${ }^{9}$ 
On the otherhand, for $\left|\omega-\bar{\omega}_{d E}\right| \sim\left|\omega_{b E}\right|$, the $l \neq 0$ terms dominate. In fact, similar to the circulating particles, we have two $\theta$ scales; i.e., $\theta_{0} \sim O\left(\theta_{T}\right) \lesssim 1$ and $\theta_{k} \sim O\left(1 / k_{\theta} \rho_{b}\right) \sim \epsilon^{-1 / 2}$ with $\rho_{b}=\Omega_{d} / \omega_{b}$. Carrying out straightforward algebra, we then have

$$
\begin{aligned}
\delta W_{K T} & \simeq \frac{8 \pi^{2} e^{2} q R_{0} B_{0} \theta_{T}^{2}}{m c^{2}} \int d \mathcal{E} \int d \mu Q F_{0} \Omega_{d}^{2} \tau_{b} \\
& \times \sum_{l \neq 0} \sum_{p} \frac{p^{2} J_{p}^{2}\left(\lambda_{b}\right)}{\lambda_{b}^{2}} \frac{\bar{\omega}_{d}-\omega}{\left(\bar{\omega}_{d}-\omega\right)^{2}-p^{2} \omega_{b}^{2}}
\end{aligned}
$$

Here, $\lambda_{b}=\theta_{T} \hat{k}_{\perp} \rho_{b}$. Again, using Padé's approximant for the Bessel functions and replacing $\sum_{l} \neq 0$ by integration, Eq (37) becomes

$$
\delta W_{K^{\prime} T} \simeq \frac{\pi^{2} e^{2} q R_{0} B_{0} \theta_{T}^{2}}{m c^{2} s} \int d \mathcal{E} \int d \mu Q F_{0} \Omega_{d^{2} \tau_{b}} \frac{1}{\Delta_{b}\left(1+\Delta_{b}^{2}\right)^{3 / 2}} \frac{\bar{\omega}_{d}-\omega}{\left(\bar{\omega}_{d}-\omega\right)^{2}-\omega_{b}^{2}}
$$

Here, $\Delta_{b}^{2}=\lambda_{b}^{2} / 8$. Note also that, in Eq. (38), we have only kept $p=$ \pm 1 terms; which, according to discussions similar to the case of circulating particles, are generally the dominant ones.

Within our orderings, $\omega_{* p i}<\omega_{d E}, \omega_{b E}, \omega_{t E}<\omega_{* E}$, we get $\delta W_{K_{r}}>0$ for $\omega \lesssim \omega_{\text {spi }}$ from Eqs. (32), (36), and (38). The causality condition Eq. (14) thus tells us the MHD gap mode can only be excited when the system is ideal MHD unstable $\left(\delta W_{f}<0\right)$. Meanwhile, from Eq. (17) and Eq. (20), we recall that the existence of the unstable energetic-particle continuum mode requires $\partial \delta W_{K_{r}} / \partial \omega_{r}<0$, and $\delta W_{K_{r}}<0$ for ideal MHD stable plasmas $\left(\delta W_{\rho}>\right.$ $0)$. By investigating Eqs. (32), (36), and (38), we find that, given proper distribution functions, both conditions can indeed be satisfied for circulating as well as trapped energetic particles. Generally speaking, it implies that the energetic-particle continuum modes have characteristic frequencies $\omega_{r} \sim \omega_{t E}$ for circulating particles, and $\omega_{r} \sim \bar{\omega}_{d E}$ or $\omega_{b E}$ for trapped particles. Specific results pertinent to a slowing-down beam ion distribution will be presented in the next section.

The growth rate of energetic particle driven instability is proportional to $\delta W_{K_{i}}$; which is generally positive definite for $\omega_{-E}>\omega_{r}$. From, Eqs. (32), (36) and (38), $\delta W_{K i}$ is proportion to the $\beta_{E}$ and its radial gradient. In 
previous analyses, ${ }^{11,12} k_{\theta} \rho_{E} \lesssim|\Omega| \sim \epsilon$, one has the results with negligible urbit-size effecis and $\delta W_{K_{i}} \propto k_{\theta} \rho_{E}$ (due to $\omega_{* E}$ ). In the present analysis, $|\Omega| \lesssim k_{\theta} \rho_{E} \lesssim O(1)$, the finite-size orbit effects are clearly exhibited by the $\Delta$ and $\Delta_{b}$ factors in, respectively, Eqs. (32) and (38). Note that these factors plus $Q F_{0}$ render $\delta W_{K}$ weakly dependent on $k_{\theta}$. Finally, we note that for $k_{\theta} \rho_{E}, k_{\theta} \rho_{b E}>1, \delta W_{K} s$ decay asymptotically as $\left(k_{\theta} \rho_{E}\right)^{-3}$ or $\left(k_{\theta} \rho_{b E}\right)^{-3}$. This property can also be readily obtained from the large argument expansions of the Bessel functions.

\section{Slowing-Down Beam Ion Distribution Function}

In this Section, we shall derive the specific expressions for the growth rates of $\mathrm{KBM}$ using the following slowing-down beam ion distribution function:

$$
F_{o E}=\frac{B_{0} \beta_{E}}{2^{5} \sqrt{2} \pi^{2} m \mathcal{E}_{m}} \frac{\sqrt{1-\alpha_{0} B_{0}}}{1-\alpha_{0} B_{0} / 2} \mathcal{E}^{-3 / 2} \delta\left(\alpha-\alpha_{0}\right) ;
$$

where $\alpha=\mu / \mathcal{E}$ is the pitch angle, $B_{0}$ is the ambient magnetic flux at magnetic axis, and $\mathcal{E} \leqslant \mathcal{E}_{m}$ with $\mathcal{E}_{m}$ being the maximum beam energy.

\section{A. Circulating Particles}

Substituting Eq. (39) into Eq. (32), we find

$$
\delta W_{K U r} \simeq \frac{\pi}{2^{3 / 2} s} \alpha_{E} \frac{\omega_{r}}{\omega_{t m}}\left[1-\frac{\omega_{r}}{2 \omega_{t m}} \ln \left|\frac{\omega_{r}+\omega_{t m}}{\omega_{r}-\omega_{t m}}\right|\right],
$$

and, for $\left|\omega_{r}\right|<\omega_{t m}$,

$$
\delta W_{K U i} \simeq \frac{\pi^{2}}{2^{5 / 2} s} \alpha_{E} \frac{\omega_{r}^{2}}{\omega_{t m}^{2}}
$$

where $\alpha_{E} \equiv q^{2} R_{0} \beta_{E}^{\prime}$, and the subscript " $m$ " indicates that the parameters are evaluated at $\mathcal{E}=\mathcal{E}_{\mathrm{m}}$.

(i) For the MHD gap mode, Eqs. (22) and (23) become, denoting $\delta W_{r}=$ $\delta W_{J}+\delta W_{K_{r}}$,

$$
\omega_{r} \simeq \frac{\omega_{* p i}+\left[\omega_{-p i}^{2}-4 \omega_{A}^{2}\left(\delta W_{r}^{2}-\delta W_{K i}^{2}\right)\right]^{1 / 2}}{2}
$$


and

$$
\gamma \simeq \alpha_{E} \frac{\pi^{2}}{2^{3 / 2} s} \frac{\omega_{r}^{2} \omega_{A}^{2}}{\omega_{t m}^{2}} \frac{\left(-\delta W_{r}\right)}{\omega_{* p i}} .
$$

$\gamma$, thus, increases with $k_{\theta}$ and is limited by $k_{\theta} \rho_{m}, k_{\theta} \rho_{d m} \lesssim 1$. Note the causality constraint $\delta W_{r}<0$ becomes, approximately,

$$
\delta W_{f}+\alpha_{E} \frac{\pi}{2^{3 / 2} s} \frac{\omega_{r}}{\omega_{t m}} \simeq \delta W_{f}<0 .
$$

Equation (44), thus, reconfirms the observation that the MHD gap mode is unstable only if the plasma is ideal MHD unstable $\left(\delta W_{f}<0\right)$.

(ii) For the energetic-particle continuum mode, instabilities can occur even if ideal MHD is stable $\left(\delta W_{f} \geqslant 0\right)$. Taking $\delta W_{f} \simeq 0$, we find from Eqs. (17) and (40) that at the instability threshold

$$
\omega_{r} \simeq 0.834 \omega_{t m} .
$$

The threshold condition, meanwhile, is given by Eqs. (19) and (41); i.e.,

$$
\alpha_{E C} \simeq 0.57 s \omega_{t m}^{2} / \omega_{A} \omega_{r}
$$

Near the threshold, the growth rate is

$$
\frac{\gamma}{\omega_{r}} \simeq \frac{\pi}{2}\left(\frac{\alpha_{E}}{\alpha_{E C}}-1\right)\left[\ln \left|\frac{\omega_{t m}+\omega_{r}}{\omega_{t m}-\omega_{r}}\right|+\frac{\omega_{t m}}{\omega_{r}} \frac{\left(2 \omega_{r}^{2}-\omega_{t m}^{2}\right)}{\left(\omega_{t m}^{2}-\omega_{r}^{2}\right)}\right]^{-1} .
$$

From Eqs. (45) to (47), we note $\alpha_{E C}$ and $\gamma$ depends weakly on $k_{\theta}$. In fact, referring to Eq. (32), it can be shown that $\gamma$ is broadly peaked about $k_{\theta} \rho_{d m} \sim$ $\epsilon_{E}^{1 / 3}$, where $\epsilon_{E} \equiv L_{p E} / R_{\circ}$ and $L_{p E}$ is the scale length of the energetic-particle pressure.

\section{B. Trapped Particles}

We will use aga: 1 the slowing-down beam ion distribution function, Eq. (39), for the trapped particle calculations and ignore the finite Larmor-radius effects of $J_{0}^{2}\left(\lambda_{\rho}\right)$; but keep the finite banana-width (finite $\lambda_{b}$ ) contributions. Corresponding expressions of $\delta W_{K T}$ can then be derived using Eqs. (36) and (38) for, respectively, $|\omega| \sim \bar{\omega}_{d E}<\omega_{b E}$ and $|\omega| \sim \omega_{b E}>\bar{\omega}_{d E}$. Note the 
case with $\bar{\omega}_{d E} \gtrsim \omega_{b E}$ (i.e., precessional drift-bounce resonance) is effectively suppressed by the finite- $\lambda_{b}$ effects.

(i.) For $|\omega| \sim\left|\bar{\omega}_{d E}\right|<\left|\omega_{b E}\right|$, the mode is driven by the precessional drift resonance. We have

$$
\delta W_{K T r} \simeq \frac{\pi}{\sqrt{2}} \alpha_{E}\left[1-\frac{\omega_{r}}{\bar{\omega}_{d m}} \ln \left|\frac{\omega_{r}}{\bar{\omega}_{d m}-\omega_{r}}\right|\right],
$$

and

$$
\delta W_{K T i} \simeq \frac{\pi^{2}}{\sqrt{2}} \frac{\omega_{r}}{\bar{\omega}_{d m}} \alpha_{E}
$$

(i.a) The MHD gap mode can be excited when

$$
\delta W_{r} \equiv \delta W_{f}+\delta W_{K T r} \simeq \delta W_{f C}<0 .
$$

Here, $\delta W_{f C}$ denotes ideal MHD $\delta W$ with only the core component contribution. While $\omega_{r}$ is given by Eq. (42), the corresponding growth rate is

$$
\frac{\gamma}{\omega_{r}} \simeq \alpha_{E} \sqrt{2} \pi^{2} \frac{\omega_{A}^{2}}{\bar{\omega}_{d m}} \frac{\left(-\delta W_{r}\right)}{\omega_{* p i}}
$$

$\gamma$, thus, scales inversely with $\omega_{* p i}$ or $k_{\theta}$. Equation (51), however, assumes $\omega_{\text {spi }} \gtrsim 2\left|\delta W_{r}\right| \omega_{A}$; i.e., the purely ideal ballooning mode is kinetically stabilized by $\omega_{* p i}$.

(i.b) As to the energetic-particle continuum mode, the instability, again, can occur even if $\delta W_{f} \geqslant 0$. Taking $\delta W_{f} \simeq 0$, we have

$$
\omega_{r} \simeq 0.78 \bar{\omega}_{d m} .
$$

The corresponding threshold value of $\alpha_{E}$ is then given by

$$
\alpha_{E C} \simeq 0.14 \bar{\omega}_{d m} / \omega_{A} .
$$

Meanwhile, near the threshold, the growth rate is

$$
\frac{\gamma}{\omega_{r}} \simeq \pi\left(\frac{\alpha_{E}}{\alpha_{E C}}-1\right)\left[\ln \left|\frac{\omega_{r}}{\bar{\omega}_{d m}-\omega_{r}}\right|+\frac{\bar{\omega}_{d m}}{\bar{\omega}_{d m}-\omega_{r}}\right]^{-1} .
$$

Thus, both $\alpha_{E C}$ and $\gamma$ scale with $k_{\theta}$ for $k_{\theta} \rho_{b m} \lesssim 1$. 
(ii) For $|\omega| \sim\left|\omega_{b E}\right|>\left|\bar{\omega}_{d E}\right|$, the mode is driven by the bounce resonance. We have

$$
\delta W_{K T r} \simeq \frac{\pi}{2} \frac{\theta_{T}}{s} \alpha_{E} \frac{\omega_{r}}{\omega_{b m}}\left[1-\frac{\omega_{r}}{2 \omega_{b m}} \ln \left|\frac{\omega_{b m}+\omega_{r}}{\omega_{b m}-\omega_{r}}\right|\right],
$$

and, for $\left|\omega_{r}\right|<\omega_{b m}$

$$
\delta W_{K T i} \simeq \frac{\pi^{2} \theta_{T}}{4 s} \frac{\omega_{r}^{2}}{\omega_{b m}^{2}} \alpha_{E}
$$

We note that Eqs. (55) and (56) have the same functional dependencies as those of Eqs. (40) and (41) for the circulating particles, and, therefore, the stability properties can also be expected to be similar.

(ii.a) The MHD gap mode, again, can be excited when

$$
\delta W_{r} \simeq \delta W_{f}+\frac{\pi}{2} \frac{\theta_{T}}{s} \alpha_{E} \frac{\omega_{* p i}}{\omega_{b m}} \simeq \delta W_{f}<0 ;
$$

i.e., only when $\delta W_{f}<0$. The corresponding growth rate is then

$$
\gamma \simeq \alpha_{E} \frac{\pi^{2}}{2} \frac{\theta_{T}}{s}\left(\frac{\omega_{r}^{\prime} \omega_{A}}{\omega_{b m}}\right)^{2}\left(-\frac{\delta W_{r}}{\omega_{* p i}}\right) .
$$

(ii.b) Similar to the case of circulating particles, the energetic-particle continuum mode can be destabilized when $\alpha_{E}$ exceeds the threshold value even if the plasma is ideal MHD stable $\left(\delta W_{f} \geq 0\right)$. In this case, we have for $\delta W_{f} \simeq 0$,

$$
\omega_{r} \simeq 0.834 \omega_{b m}
$$

Meanwhile,

$$
\alpha_{E C} \simeq 0.4 \frac{s}{\theta_{T}} \frac{\omega_{b m}^{2}}{\omega_{r} \omega_{A}}
$$

and, near the threshold,

$$
\frac{\gamma}{\omega_{r}} \simeq \frac{\pi}{2}\left(\frac{\alpha_{E}}{\alpha_{E C}}-1\right)\left[\ln \left|\frac{\omega_{b m}+\omega_{r}}{\omega_{b m}-\omega_{r}}\right|+\frac{\omega_{b m}\left(2 \omega_{r}^{2}-\omega_{b m}^{2}\right)}{\omega_{r}\left(\omega_{b m}^{2}-\omega_{r}^{2}\right)}\right]^{-1} .
$$

Note that, similar to the case of circulating particles, the growth rate of the energetic-particle continuum mode is broadley peaked about $k_{\theta} \rho_{b m} \sim \epsilon_{E}^{1 / 3}$ and depends weakly on $k_{\theta}$. 
It is instructive to assess the relative importance between the precessional drift and bounce resonances. For the MHD gap mode, one can readily see from. Eqs. (51) and (58) that the precessional drift resonance, Eq. (51), dominates. As to the energetic particles continuum mode, Eqs. (53) and (60) clearly indicate that the precessional drift resonance has a lower threshold for $k_{\theta} \rho_{b m} \lesssim 1$. For high $\alpha_{E}$ values, such that continuum modes corresponding to both resonances are excited with comparable growth rates, the precessionaldrift mode tends to have higher $k_{\theta}, k_{\theta} \rho_{b m} \sim 1$; while the bounce mode will have $k_{\theta} \rho_{b m} \sim \epsilon_{E}^{1 / 3}$.

\section{Summary and Discussions}

In the the present work, we have carried out a matched asymptotic analysis and derived an analytic dispersion relation for the kinetic ballooning modes (KBM) resonantly excited by energetic ions/alpha particles in tokamaks. Our analysis contains effects due to finite-size orbits of both circulating and 'rapped energetic particles; such as Larmor radii, magnetic drift orbits and banana widths, which render energetic particle contributions effective only in the ideal region instead of the extended inertial region as in the case of negligible orbit sizes. The dispersion relation, furthermore, include various wave-particle resonances and is of the generic "fishbone" form.

We have analyzed this KBM dispersion relation and found that it exhibits two types of unstable modes. One is the MHD gap mode with $\omega_{\text {*pi }} / 2 \lesssim \omega_{r} \lesssim$ $\omega_{* p i}$ which exists only when the tokamak plasma is ideal MHD unstable, i.e., $\delta W_{f}<0$. The other is the energetic-particle continuum mode with $\omega_{r}>\omega_{* p i} ;$ that is, $\omega_{r}$ lies inside the continuum. Typically, $\omega_{r}$ corresponds to the characteristic energetic particle frequencies such as $\bar{\omega}_{d E}, \omega_{b E}$ or $\omega_{t E}$. The energetic-particle continuum mode can be excited even if $\delta W_{\rho}>0$; but there is a threshold in $\alpha_{E}$ due to the resonant continuum damping.

To illustrate these properties more explictly, we have further analyzed the dispersion relation using a slowing-down beam ion distribution function and derive specific expressions of $\omega_{r}, \gamma$, and threshold $\alpha_{E}$ (if applicable) for both types of modes as well as circulating and trapped particles.

Several approximations have been made in this paper in order to sir.uplify the analyses and, thereby, illuminate the physics. We expect straightforward extensions of the present work to more realistic tokamka equilibria and/or 
to include additional effects such as core-component damping, global continuum damping, etc. The qualitative features of the instability properties are, however, expected to be similar is those obtained here. 


\section{Appendix A. Derivation of Equation (33)}

Note that Eq. (31)can be written as

$$
J_{0}\left(\lambda_{\rho}\right) 2 J_{1}\left(\lambda_{d}\right) / \lambda_{d} \simeq\left(1+\Delta^{2} k_{\perp}^{2} / k_{\theta}^{2}\right)^{-1}=\left(1+\Delta^{2}\right)^{-1}\left(1+\Lambda^{2} \theta^{2}\right)^{-1}
$$

where $\Delta^{2} \equiv k_{\theta}^{2}\left(\rho^{2}+\rho_{d}^{2} / 2\right) / 4$ and $\Lambda^{2} \equiv s^{2} \Delta^{2} /\left(1+\Delta^{2}\right)$. Fourier transforming Eq. (27) to $z-$ space by $\int_{-\infty}^{\infty} e^{-i z \eta} d \eta$, we have

$$
i\left(1-\Lambda^{2} \partial_{z}^{2}\right)\left(\omega_{t} z-\omega\right) \delta \hat{G}_{d}=\pi \frac{e}{m} \frac{Q F_{0}}{\left(1+\Delta^{2}\right)} \frac{\Omega_{d}}{\omega}[\delta(z-1)-\delta(z+1)],
$$

and the solution is

$$
\delta \hat{G} \simeq \frac{i \pi}{2\left(\omega_{t} z-\omega\right)} \frac{e}{m} \frac{Q F_{0} \Omega_{d}}{\omega\left(1+\Delta^{2}\right)} \frac{1}{\Lambda}\left[e^{-|z+1| / \Lambda}-e^{-|z-1| / \Lambda}\right] .
$$

Similarly, we have, from Eq. (26),

$$
\delta \hat{S}_{d}^{*} \simeq-\frac{i \pi \Omega_{d}}{2\left(1+\Delta^{2}\right) \Lambda}\left[e^{-|z+1| / \Lambda}-e^{-|z-1| / \Lambda}\right] .
$$

Substituting Eqs. (A3) and Eqs. (A4) into Eq. (25) along with Parseval's theorem, we find

$$
\delta W_{K_{u}}=\frac{\pi}{4} \frac{e^{2} \cdot i^{2} R_{0}^{2}}{m c^{2}}\left\langle\frac{Q F_{0} \Omega_{d}^{2}}{\left(1+\Delta^{2}\right)^{2} \Lambda^{2}} \int_{-\infty}^{\infty} d z \frac{1}{\omega_{t} z-\omega}\left[e^{-|z-1| / \Lambda}-e^{-|z+1| / \Lambda}\right]^{2}\right\rangle .
$$

In deriving Eq. (A5), we have noted that $|\Lambda| \sim \epsilon^{1 / 2}<1$, and thus, the integrand is peaked at $z= \pm 1$. Letting $x=2|z \pm 1| / \Lambda$ in Eq. (A5), we then derive Eq. (33).

\section{Acknowledgements}

The authors would like to acknowledge useful discussion with Dr. G.Y. Fu and Prof. T.H. Stix. One of the authors (S.T. Tsai) is grateful for the hospitality of the Princeton Plasma Physics Laboratory during the time this work was performed. Work supported by U.S. Department of Energy Contract No. DE-AC02-76-CHO3073 and NSFC. 


\section{References}

${ }^{1}$ K. McGuire, et al. Phys. Rev. Lett. 50, 89 (1983).

${ }^{2}$ W.W. Heidbrink et al. Phys. Rev. Lett. 57, 835 (1986).

${ }^{3}$ K.-L. Wong et al. Phys. Rev. Lett. 66, 1874 (1991).

${ }^{4}$ M.N. Rosenbluth, S.T. Tsai, J.W. Van Dam and M.G. Engquist, Phys. Rev. Lett. 51, 1969 (1983).

${ }^{5}$ R.J. Hastie, Y.P. Chen, F.J. Ke, S.T. Tsai, and L. Chen, Chinese Phys. Lett. 4, 561 (1987).

${ }^{6}$ L.J. Zheng and S.T. Tsai, Phys. Fluids B 4, 1416 and 3329 (1992).

${ }^{7}$ L. Chen, R.B. White, and M.N. Rosenbluth, Phys. Rev. Lett. 52, 1122 (1984).

${ }^{8}$ R.B. White, L. Chen, F. Romanelli, and R. Hay, Phys. Fluids 28, 278 (1985).

${ }^{9} \mathrm{~J}$. Weiland and L. Chen, Phys. Fluids 28, 1399 (1985).

${ }^{10} \mathrm{H}$. Biglari and L. Chen, Phys. Fluids 29, 1760 (1986).

${ }^{11}$ B. Coppi and F. Porcelli, Phys. Rev. Lett. 57, 2277 (1986).

${ }^{12} \mathrm{~L}$. Chen, in Theory of Fusion Plasmas, edited by J. Vaclavik, F. Troyon and E. Sindoni (Assoc. EURATOM, Bologna, 1988), p. 327.

${ }^{13} \mathrm{H}$. Biglari and L. Chen, Phys. Rev. Lett. 67, 3681 (1991).

${ }^{14}$ H.L. Berk, B.N. Breizman, and H. Ye, Phys. Lett. A, 162, 475 (1992).

${ }^{15}$ G.Y. Fu and C.Z. Cheng, Princeton Plasma Physics Laboratory Report 2852 (1992).

${ }^{16}$ J.W. Connor, R.J. Hastie and J.B. Taylor, Phys. Rev. Lett. 40, 396 (1978).

${ }^{17}$ L. Chen and S.T. Tsai, Plasma Phys. 25, 349 (1983). 
Dr. F. Peotoni, Univ. of Wollongong, AUSTRALIA

Prot. M.H. Brennan, Univ. of Sydney, AUSTRALIA

Plasma Posearch LD., Austrelien Nat. Univ., AUSTRALIA

Prof. I.P. Jones, Finders Univ, AUSTRALIA

Prof. F. Cep, Inat tor Theoretical Physics, AUSTRIA

Prof. M. Hoindior, Instivt for Theoretische Phyeik, AUSTRIA

Prof. M. Gooseens, Astronomiech InstituUt, BELGIUM

Ecolo Royedo Mtituira, Lob. do Phy. Plasmas, BELGIUM

Commiscion-Europaen, DG. XII-Fusion Proo., BELGIUM

Prof. R. Bouciqus, Rikeuniverituit Gent, BELGIUM

Dr. P.H. Sakenaka, Instivio Fivica, BRUZIL

Instius Neciond Do Posquiess Especisie-INPE, BRUZIL

Documents Orion, Alomic Energy of Cenada Ld., CAMADA

Or. M.P. Bactynaki, MPB Tectrologise, Inc., CAMLDA

Dr. H.M. Skercoard, Univ. of Sackatchewen, CANADA

Prof. J. Teichmann, Univ. of Montreal, CANADA

Prof. S.R. Srectiveen, Univ. of Celoary, CANAOA

Prot. T.W. Johnetion, INRS-Energio, CANADA

Dr. R. Botion, Contre canadien do tusion meanotique, CANADA

Dr. C.R. James., Univ. of Nborta, CANADA

Dr. P. Lukte, Komenatcho Universite. CZECHOSLOVAKIA

The Librarien, Cuthem Loboratory, ENGLAND

Librery, R61, Ruthertord Appletion Leboratory. ENGLAND

Mrs. S.A. Hutchimeon, JET Librery, ENGLAND

Dr. S.C. Shama, Univ. of South Pecific, FIJI ISLANDS

P. Menomen, Univ. of Helsinti, FINLAND

Prot. M.N. Buseace, Ecols Potyochnique., FRANCE

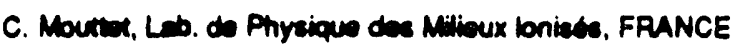

J. Radol, CENCADARACHE - Bat 506, FRANCE

Prot. E. Economou, Univ. of Crem, GAEECE

Mo. C. Pinni, Univ. of loannina, GREECE

Dr. T. Mul, Acadermy Enviogrephic Ser. HONG KONG

Proprint Librery, Hunoerien Acadermy of SC.. HUNGARY

Dr. B. Descupta, Saha inst of Nucterer Ptysics, INDIA

Dr. P. Kaw, Inat for Pleema Pacearch, INDIA

Dr. P. Pocensu, lered inst of Technology, ISRAEL

Librevien, Internaciond Comber for Theo Physice, ITALY

Miss C. Do Pab, Asecciaziono EURATOMENEA, ITALY

Dr. G. Groses, lesiuto di Fieven ded Plasma, ITALY

Prof. G. Rostenoni, lesturo Gas lonizzeid Del Cnr, ITALY

Dr. H. Yemes, Toehibe Ras a Dovel Conter, JAPAN
Prot. I. Kumakemi, Hiroshima Univ., JAPAN

Prof. K. Nishikawe, Hiroshima Univ., LAPAN

Oirector, Japen Atomic Eneroy Research Inst. JAPAN

Prof. S. Itoh, Kyushu Univ., MAPAN

Reserech info. Cr., Nationd Instit, for Fusion Science, JAPAN

Prof. S. Tenake, Kyoto Univ., JAPAN

Librery, Kyoto Univ., JAPAN

Prof. N. Inow, Univ. of Towyo, LAPAN

Secretary, Plema Section, Electrolechnical Lab., JAPAN

S. Mori, Technied Acticor, MEPH, MAPAN

Dr. O. Mined, Kumemoso inst of Technology, JAPAN

J. Hyeon-Sook, Korea Atomic Energy Rasearch Inst, KOREA

D.I. Chai, Tho Korea Adv. Inet of Sai. \& Tech., KOAEA

Prot. B.S. Liby, Univ. of Weikato, NEW ZEALAND

Inst of Physice, Chinese Aced Sa PEOPLE'S REP. OF CHINA

Library, Inat of Plasm Physics, PEOPLE'S REP OF CHINA

Teinghua Univ. LibreY, PEOPLE'S REPUBLIC OF CHINA

Z Li, S.W. Inst Phytic, PEOPLE'S REPUBUC OF CHINA

Prot. J.A.C. Cebrd, Imsturto Superior Tecnico, PORTUGAL

Dr. O. Potrus, al I Cuza Univ., Romania

Dr. J. do Vilien, Fusion Sudios, AEC, S. AFRICA

Prot. M.A. Hemberg. Univ. of Netal, S. AFRICA

Prol. D.E. Kin, Pohang Inat. of Sci. Tech., SO. KOAEA

Prot. C.I.E.MA.T, Fuaion Divizion Library. SPAIN

Dr. L SEmilo, Univ. of UMEA, SWEDEN

Lbrey, Royd inct of Tectnology, SWEDEN

Prot. H. Witheineon, Chedmen Univ. of Tech., SWEDEN

Cons Phys. Des Phamas, Ecols Pohytech, SWITZERLAND

Bibliothock, Ina. Voor Piesme-Fycica, THE NETHERLANDS

Aset. Prot. Dr. S. Cadxt, Midde Eest Tech. Univ., TUAKEY

Or. V.A. Gulthin,Sa. Res. Inst Electophys.I Apparatus, USSR

Dr. D.D. Ayutov, Siberien Enanch of Acadamy of Sa., USSR

Dr. G.A. Eicen, I.V. Kurchatov Inse, USSA

Librerien, The Ukr.SSA Acodemy of Scionces, USSR

Dr. LM. Kowiathmykh, int. of Gonord Physics, USSR

Kemtorchungsantepe GmbH, Zentrabibliothek, W. GERMANY

Bibliothok, Inst. FOr Pinsmalorchung, W. GERMANY

Prof. K. Schinder. Ruhr-Univereity Bochum, W. GERMANY

Dr. F. Wegner, (ASOEX), MaX-Planck-Institut, W. GERMANY

Libriten, Max-Plenck-Instient, W. GEAMANY

Prof. R.K. Janw, Inat of Plycics, VUcosLaVIA 

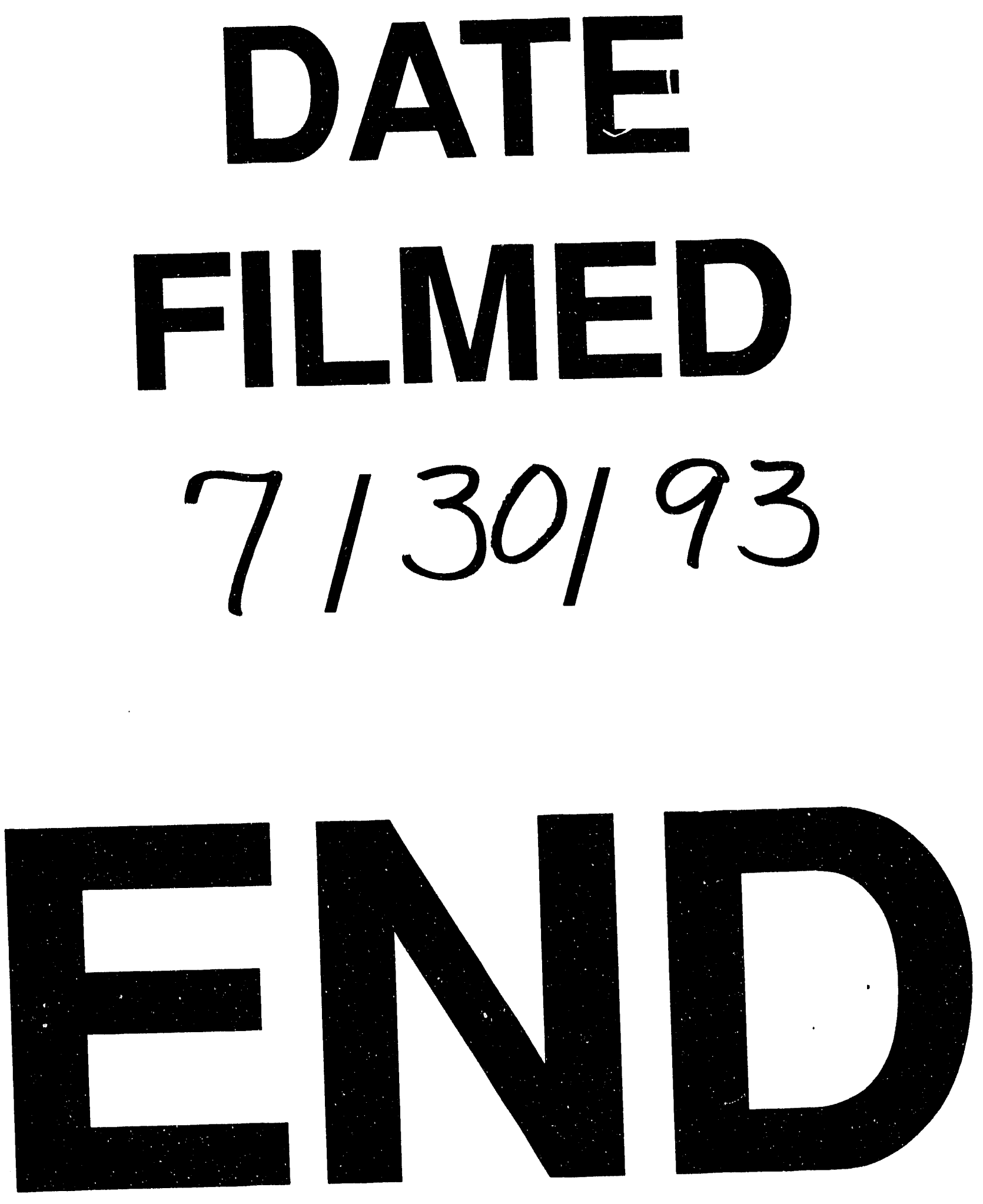
